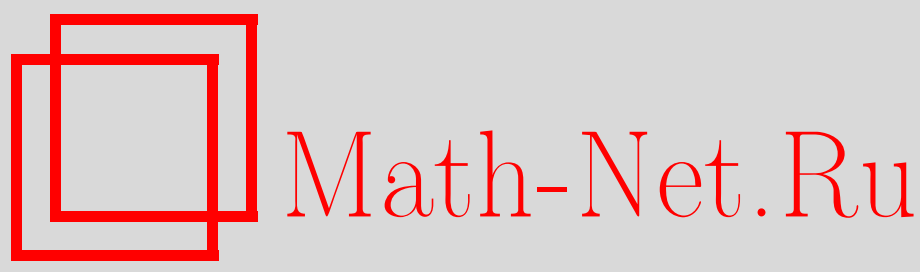

А. С. Белов, Порядковые оценки модуля изменения суммы лакунарного тригонометрического ряда, Матем. сб., 1998, том 189, номер 5, 3-20

DOI: https://doi.org/10.4213/sm317

Использование Общероссийского математического портала Math-Net.Ru подразумевает, что вы прочитали и согласны с пользовательским соглашением

http://www . mathnet.ru/rus/agreement

Параметры загрузки:

IP: 52.90 .164 .192

26 апреля 2023 г., $14: 25: 52$ 
УДК 517.5

\author{
А.С. Белов
}

\title{
Порядковые оценки модуля изменения суммы лакунарного тригонометрического ряда
}

\begin{abstract}
В статье находятся порядковые оценки для модуля изменения и усредненного модуля суммы лакунарного тригонометрического ряда через его коэффициенты.

Эти интересные глобальные характеристики функции и их применения изучались в работах 3. А. Чантурия, Е. П. Долженко, Е. А. Севастьянова, Б. Сендова, В. Попова и других. Поскольку в теории функций в качестве примера функции с теми или иными свойствами достаточно часто используется сумма лакунарного тригонометрического ряда, то полезно знать о ней как можно больше и, в частности, такие ее глобальные характеристики, как модуль изменения и усредненный модуль.

Приводятся также необходимые и достаточные условия принадлежности суммы лакунарного тригонометрического ряда некоторьм классам функций, определяемьм через указанные характеристики.

Библиограбия: 11 названий.
\end{abstract}

\section{$\S 1$. Введение}

Моему учителю П. Л. Ульянову посвящается эта работа в связи с его семидесятилетием.

Пусть действительная функция $f$ непрерывна на сегменте $I=[\alpha, \beta], \alpha<\beta$. Для каждого натурального числа $n$ определим величину

$$
\nu_{f}(n)_{I}=\sup \sum_{k=1}^{n}\left|f\left(\beta_{k}\right)-f\left(\alpha_{k}\right)\right|
$$

где верхняя грань берется по всевозможным наборам непересекающихся интервалов $\left\{\left(\alpha_{k}, \beta_{k}\right)\right\}_{k=1}^{n}$, лежаших в $I$. Положим $\nu_{f}(0)_{I}=0$. Последовательность $\left\{\nu_{f}(n)_{I}\right\}_{n=0}^{\infty}$, следуя терминологии 3. А. Чантурия [1], будем называть модулем изменения функции $f$ на сегменте $I$. Для любого сегмента $J \subset I$ пусть

$$
\Omega_{f}(J)=\max _{x \in J} f(x)-\min _{x \in J} f(x)=\max \left\{\left|f\left(x_{1}\right)-f\left(x_{2}\right)\right|: x_{1}, x_{2} \in J\right\} .
$$

Тогда при любом натуральном $n$ справедливо равенство

$$
\nu_{f}(n)_{I}=\sup \sum_{k=1}^{n} \Omega_{f}\left(\left[x_{k-1}, x_{k}\right]\right)
$$

Работа выполнена при финансовой поддержке Российского фонда фундаментальных исследований (грант № 96-01-00094). 
где верхняя грань берется по всем точкам $\alpha=x_{0} \leqslant x_{2} \leqslant \cdots \leqslant x_{n}=\beta$. Модуль изменения является интересной глобальной характеристикой функции $f$. Подробнее о свойствах модуля изменения см. работы [1], [2]. Для наших целей достаточно знать только следующие простейшие свойства модуля изменения, которые легко вытекают из (1) и (2): $\nu_{f}(n-1)_{I} \leqslant \nu_{f}(n)_{I}$ и $n^{-1} \nu_{f}(n)_{I} \geqslant(n+1)^{-1} \nu_{f}(n+1)_{I}$ при всех натуральных $n$.

Пусть $\Delta=|I|=\beta-\alpha$ - длина сегмента $I, n-$ натуральное число и $\left\{\left(\alpha_{k}, \beta_{k}\right)\right\}_{k=1}^{n}$ - произвольный набор непересекающихся интервалов, лежащих в $I$. Для каждого $k=1, \ldots, n$ обозначим через $m_{k}$ наибольшее целое неотрицательное число такое, что $m_{k} \Delta \leqslant n\left(\beta_{k}-\alpha_{k}\right)$. Тогда

$$
\begin{aligned}
\sum_{k=1}^{n}\left|f\left(\beta_{k}\right)-f\left(\alpha_{k}\right)\right| \leqslant & \sum_{k=1}^{n}\left|f\left(\beta_{k}\right)-f\left(\alpha_{k}+m_{k} \frac{\Delta}{n}\right)\right| \\
& +\sum_{k=1}^{n} \sum_{j=1}^{m_{k}}\left|f\left(\alpha_{k}+j \frac{\Delta}{n}\right)-f\left(\alpha_{k}+(j-1) \frac{\Delta}{n}\right)\right|
\end{aligned}
$$

Поскольку $\sum_{k=1}^{n} m_{k} \leqslant \frac{n}{\Delta} \sum_{k=1}^{n}\left(\beta_{k}-\alpha_{k}\right) \leqslant n$, то в последней двойной сумме не более $n$ слагаемых. Следовательно, для любого натурального $n$ верно неравенство $\nu_{f}(n)_{I} \leqslant 2 \sup \sum_{k=1}^{n}\left|f\left(\beta_{k}\right)-f\left(\alpha_{k}\right)\right|$, где верхняя грань берется по всем наборам непересекающихся интервалов $\left\{\left(\alpha_{k}, \beta_{k}\right)\right\}_{k=1}^{n}$, которые лежат в $I$ и имеют длину $\beta_{k}-\alpha_{k} \leqslant|I| / n$ при всех $k=1, \ldots, n$. В частности (см. также [1]),

$$
\nu_{f}(n)_{I} \leqslant 2 n \omega\left(\frac{|I|}{n} ; f\right)_{I} \text { при } n=1,2, \ldots,
$$

где $\omega(\delta ; f)_{I}=\sup \left\{\left|f\left(x_{1}\right)-f\left(x_{2}\right)\right|: x_{1}, x_{2} \in I,\left|x_{1}-x_{2}\right| \leqslant \delta\right\}, \delta \geqslant 0,-$ модуль непрерывности функции $f$ на сегменте $I$. При всех $\delta \geqslant 0$ положим

$$
\tau_{f}(\delta)_{I}=\frac{1}{|I|} \int_{I} \Omega_{f}\left(\left[x-\frac{\delta}{2}, x+\frac{\delta}{2}\right] \cap I\right) d x .
$$

Функцию $\tau_{f}(\delta)_{I}$ будем, для краткости, называть просто усредненным модулем функции $f$. О свойствах этой глобальной характеристики функции $f$ см. работы $[3],[4]$. Нам достаточно знать, что $\tau_{f}(\delta)_{I}$ - неотрицательная, неубывающая непрерывная функция на $[0, \infty)$ такая, что $\tau_{f}(0)_{I}=0, \tau_{f}(\delta)_{I}=\tau_{f}(2|I|)_{I}=\Omega_{f}(I)$ при $\delta \geqslant 2|I|$ и $\tau_{f}\left(\delta_{1}+\delta_{2}\right)_{I} \leqslant \tau_{f}\left(\delta_{1}\right)_{I}+\tau_{f}\left(\delta_{2}\right)_{I}$ при всех $\delta_{1} \geqslant 0$ и $\delta_{2} \geqslant 0$. Для каждого натурального $n$ из (2) и (4) имеем

$\tau_{f}\left(\frac{\Delta}{n}\right)_{I}=\frac{1}{\Delta} \int_{\alpha}^{\alpha+\Delta / n} \sum_{k=0}^{n-1} \Omega_{f}\left(\left[x+\frac{k \Delta}{n}-\frac{\Delta}{2 n}, x+\frac{k \Delta}{n}+\frac{\Delta}{2 n}\right] \cap I\right) d x \leqslant \frac{1}{n} \nu_{f}(n)_{I}$.

Таким образом, усредненный модуль и модуль изменения функции $f$ связаны соотношением

$$
\tau_{f}\left(\frac{|I|}{n}\right)_{I} \leqslant \frac{\nu_{f}(n)_{I}}{n} \text { при всех } n \geqslant 1 .
$$

Более подробно о связи между такими глобальными характеристиками функции $f$ как усредненный модуль и модуль изменения см. работу Е. А. Севастьянова [5], 
а об их применении - книгу Б. Сендова и В. Попова [3] и обзорную статью М. Авдиспахича [6].

Цель настоящей статьи - считая функцию $f$ суммой лакунарного тригонометрического ряда, найти порядковые оценки для модуля изменения и усредненного модуля функции $f$ через коэффициенты соответствующего лакунарного ряда. Поскольку в теории функций в качестве примера функции с теми или иными свойствами достаточно часто удается использовать сумму лакунарного тригонометрического ряда, то, на наш взгляд, важно знать и такие полезные глобальные характеристики суммы лакунарного ряда, как усредненный модуль и модуль изменения.

\section{§2. Обозначения и основные результаты}

Пусть всюду далее

$$
f(x)=a+\sum_{n=1}^{\infty} a_{n} \cos \left(\lambda_{n} x+\psi_{n}\right)
$$

где последовательность положительных чисел $\left\{\lambda_{n}\right\}_{n=1}^{\infty}$ удовлетворяет условию

$$
\frac{\lambda_{n+1}}{\lambda_{n}} \geqslant \lambda>1 \text { при всех } n=1,2, \ldots,
$$

последовательность неотрицательных чисел $\left\{a_{n}\right\}_{n=1}^{\infty}$ такова, что $\sum a_{n}<\infty$, а действительные числа $a$ и $\left\{\psi_{n}\right\}_{n=1}^{\infty}$ произвольны. Все эти условия далее всегда считаются выполненными. Для удобства будем считать, что $\lambda_{0}=0$ и $\pi / \lambda_{0}=+\infty$. Введем обозначения

$$
\begin{gathered}
N(\delta)=N \text { при } \delta \in\left[\frac{\pi}{\lambda_{N}}, \frac{\pi}{\lambda_{N-1}}\right), \quad N \geqslant 1 ; \\
R(y)=\sum_{n: \lambda_{n} \geqslant y} a_{n}, \quad T(y)=\sum_{n: \lambda_{n}<y} a_{n} \lambda_{n}, \\
U(y)=\left(\sum_{n: \lambda_{n}<y} a_{n}^{2} \lambda_{n}^{2}\right)^{1 / 2}, \quad L(y)=U(y)+y R(y) \text { при } y>0 ; \\
v_{n}=\sum_{k=1}^{n} a_{k} \lambda_{k}, \quad w_{n}=\left(\sum_{k=1}^{n} a_{k}^{2} \lambda_{k}^{2}\right)^{1 / 2} \text { при } n \geqslant 0 ; \\
a(\delta)=\sum_{n=N(\delta)}^{\infty} a_{n}=R\left(\frac{\pi}{\delta}\right) \text { при } \delta>0 ; \\
\omega_{1}(\delta)=\sum_{n=1}^{\infty} a_{n} \min \left(\lambda_{n} \delta, \pi\right) \text { при } \delta \geqslant 0 ; \\
\omega_{0}(\delta)=\sum_{n=1}^{\infty} \lambda_{n}^{-1}\left(w_{n}-w_{n-1}\right) \min \left(\lambda_{n} \delta, \pi\right) \text { при } \delta \geqslant 0 .
\end{gathered}
$$

Во всем дальнейшем изложении все приведенные вьше обозначения используются только в указанном смысле; в частности, $f(x)$ всюду обозначает сумму ряда (6). Ссылка на (8) указывает на использование некоторых из этих обозначений. 
Напомним, что модулем непрерывности называется определенная и непрерывная при $\delta \geqslant 0$ неубываюшая функция $\omega$ такая, что $\omega(0)=0$ и $\omega\left(\delta_{1}+\delta_{2}\right) \leqslant \omega\left(\delta_{1}\right)+\omega\left(\delta_{2}\right)$ при всех $\delta_{1} \geqslant 0$ и $\delta_{2} \geqslant 0$. Если к тому же функция $\omega$ выпукла вверх, то она называется выпукльм модулем непрерывности. Заметим, что при каждом натуральном $n$ функция $\min \left(\lambda_{n} \delta, \pi\right)$ является выпуклым модулем непрерывности. Очевидна также оценка

$$
\lambda_{n}^{-1}\left(w_{n}-w_{n-1}\right) \leqslant a_{n} \text { при } n \geqslant 1
$$

Поэтому функции $\omega_{1}(\delta)$ и $\omega_{0}(\delta)$ как суммы равномерно сходящихся рядов из выпуклых модулей непрерывности являются вьпуклыми модулями непрерьвности. В частности, функции $\delta^{-1} \omega_{1}(\delta)$ и $\delta^{-1} \omega_{0}(\delta)$ не возрастают на $(0, \infty)$. Следует также отметить, что $\omega_{1}(\delta)=\pi \sum_{n=1}^{\infty} a_{n}$ и $\omega_{0}(\delta)=\pi \sum_{n=1}^{\infty} \lambda_{n}^{-1}\left(w_{n}-w_{n-1}\right)$ при $\delta \geqslant \pi / \lambda_{1}$, т.е. функции $\omega_{1}$ и $\omega_{0}$ постоянны на $\left[\pi / \lambda_{1},+\infty\right)$. Очевидно, что они линейны на каждом из сегментов $\left[\pi / \lambda_{N}, \pi / \lambda_{N-1}\right]$, где $N=2,3, \ldots$. Функции $\omega_{1}$ и $\omega_{0}$ являются важными характеристиками лакунарного ряда (6).

Имеет место (см. $\S 3$ )

Лемма 1. Верны оценки:

$$
\left(\frac{2 \lambda-1}{\lambda-1}\right) \omega_{0}(\delta) \geqslant \delta L\left(\frac{\pi}{\delta}\right)=\delta U\left(\frac{\pi}{\delta}\right)+\pi a(\delta) \geqslant \omega_{0}(\delta) \quad \text { nри всех } \delta>0
$$

$u$

$$
\pi \sum_{m=n}^{\infty} \frac{w_{m}}{\lambda_{m}} \geqslant \omega_{0}\left(\pi \lambda_{n}^{-1}\right) \geqslant\left(1-\frac{1}{\lambda}\right) \pi \sum_{m=n}^{\infty} \frac{w_{m}}{\lambda_{m}} \quad \text { nрu всех } n \geqslant 1 .
$$

Полагая $\delta=\pi / y$, оценки (10) можно записать в виде

$$
\left(\frac{2 \lambda-1}{\lambda-1}\right) y \omega_{0}\left(\frac{\pi}{y}\right) \geqslant \pi L(y)=\pi U(y)+\pi y R(y) \geqslant y \omega_{0}\left(\frac{\pi}{y}\right) \text { при } y>0 .
$$

Пусть $I=[\alpha, \beta]$, где $\alpha<\beta,-$ произвольный сегмент, который в дальнейшем обычно считается фиксированньм, а его длина обозначается через $\Delta=|I|=\beta-\alpha$.

Буква $A$ всюду далее обозначает положительную достаточно большую постоянную, в каждом случае свою, зависящую только от тех параметров, которые указаны за ней в скобках.

Основным результатом этой статьи является следующая

ТЕОРема 1. Для произвольного сегмента $I,|I|>0$, справедливы оценки:

$$
\begin{gathered}
A^{-1}\left(\lambda, \lambda_{1}|I|\right) \omega_{0}(\delta) \leqslant \tau_{f}(\delta)_{I} \leqslant A\left(\lambda, \lambda_{1}|I|\right) \omega_{0}(\delta) \quad \text { npu } \quad \delta \geqslant 0, \\
\tau_{f}\left(\frac{|I|}{n}\right)_{I} \leqslant \frac{\nu_{f}(n)_{I}}{n} \leqslant A\left(\lambda, \lambda_{1}|I|\right) \tau_{f}\left(\frac{|I|}{n}\right)_{I} \quad n p u \quad n \geqslant 1, \\
A^{-1}\left(\lambda, \lambda_{1}|I|\right) n \omega_{0}\left(\frac{|I|}{n}\right) \leqslant \nu_{f}(n)_{I} \leqslant A\left(\lambda, \lambda_{1}|I|\right) n \omega_{0}\left(\frac{|I|}{n}\right) \quad \text { npu } n \geqslant 1 .
\end{gathered}
$$


Поскольку $\omega_{0}$ является выпуклым модулем непрерьвности, то

$\min \left\{1, \pi^{-1}|I|\right\} \omega_{0}\left(\frac{\pi}{n}\right) \leqslant \omega_{0}\left(\frac{|I|}{n}\right) \leqslant \max \left\{1, \pi^{-1}|I|\right\} \omega_{0}\left(\frac{\pi}{n}\right)$ при $n \geqslant 1$

Отсюда в силу $(15),(8)$ и (12) верны соотношения

$$
\nu_{f}(n)_{I} \asymp n \omega_{0}\left(\frac{\pi}{n}\right) \asymp U(n)+n R(n)=L(n) \text { при } n \geqslant 1
$$

и

$$
\tau_{f}(\delta)_{I} \asymp \omega_{0}(\delta) \text { при } \delta \geqslant 0,
$$

причем соответствующие постоянные в этих порядковых эквивалентностях зависят только от $\lambda, \lambda_{1}$ и $|I|$. Таким образом, порядок роста модуля изменения функции $f$, как и порядок убьвания усредненного модуля функции $f$, от сегмента $I$ не зависят. Напомним, что если функции $g$ и $h$ определены и неотрицательны на некотором множестве $E$ на прямой, то говорят, что функции $g$ и $h$ имеют на $E$ одинаковый порядок роста, и пишут $g \asymp h$ на $E$, если есть такие положительные постоянные $C_{1}$ и $C_{2}$, что $C_{1} g(x) \leqslant h(x) \leqslant C_{2} g(x)$ при всех $x \in E$.

При $p>0$ и $\gamma \in(1, p+1]$ будем писать $f \in \operatorname{ch}_{\gamma}^{p}$ на $I$, если $\sum n^{-\gamma}\left(\nu_{f}(n)_{I}\right)^{p}<\infty$. Из теоремы 1 довольно просто вытекает описание функций вида (6) из класса $\mathrm{ch}_{\gamma}^{p}$ и, в частности, из класса $\operatorname{ch}_{2}^{1}$.

СЛЕДСТВИЕ 1. Для произвольного сегмента $I,|I|>0$, справедливы следующие три утвержсдения.

1) $\operatorname{Eсли~} p>0$ и $\gamma \in(1, p+1)$, то $f \in \operatorname{ch}_{\gamma}^{p}$ на I тогда и только тогда, $\kappa о г \partial a$

$$
\sum a_{n}^{p} \lambda_{n}^{p+1-\gamma}<\infty
$$

2) Если $p>0$, то $f \in \operatorname{ch}_{p+1}^{p}$ на I тогда и только тогда, когда

$$
\int_{1}^{\infty} y^{-1}(R(y))^{p} d y<\infty
$$

3) $f \in \operatorname{ch}_{2}^{1}$ на I тогда и только тогда, когда

$$
\sum a_{n} \ln \lambda_{n}<\infty
$$

Теорема 1 также позволяет легко получить через обозначения (8) условия обратимости соотношения (3) для функции вида (6). 
СлЕДСТВИЕ 2. Следующие утверждения эквивалентны.

1) Существует положстельная постоянная $C_{1}$ такая, что

$$
n \omega\left(\frac{|I|}{n}, f\right)_{I} \leqslant C_{1} \nu_{f}(n)_{I} \quad \text { npu } \quad n \geqslant 1 .
$$

2) Существует положсительная постоянная $C_{2}$ такая, что

$$
\omega_{1}(\delta) \leqslant C_{2} \omega_{0}(\delta) \quad \text { npu } \delta \geqslant 0 .
$$

3) Существует положсительная постоянная $C_{3}$ такая, что

$$
v_{n} \leqslant C_{3}\left(w_{n}+\lambda_{n} \sum_{k=n}^{\infty} a_{k}\right) \quad n p u \quad n \geqslant 1
$$

4) Существует положсительная постоянная $C_{4}$ такая, что

$$
v_{n} \leqslant C_{4}\left(\max _{k \leqslant n}\left(a_{k} \lambda_{k}\right)+\lambda_{n} \sum_{k=n}^{\infty} a_{k}\right) \quad n p u \quad n \geqslant 1
$$

Из теоремы 1 также вытекает

СлЕДСТВИЕ 3. Для любой неубывающей последовательности положительньх чисел $\{\nu(n)\}_{n=1}^{\infty}$ такой, что последовательность $\{\nu(n) / n\}_{n=1}^{\infty}$ не возрастает и стремится $\kappa$ нулю, существует функция $f$ вида (6), для которой на любом сегменте $I,|I|>0$, справедливы соотношения

$$
n \omega\left(\frac{|I|}{n}, f\right)_{I} \asymp \nu_{f}(n)_{I} \asymp \nu(n) \quad n p u \quad n \geqslant 1 .
$$

Подробное доказательство сформулированных утверждений и составляет содержание этой статьи.

Нам осталось отметить, что доказательство леммы 1 и верхних оценок в (13) и (15) проведено в $\S 3 ; \S 4$ посвящен доказательству нижних оценок в (13) и (15) и оценок (14). Наконец, в $\S 5$ доказываются следствия 1-3. В конце статьи помещено замечание 1, в котором указьваются возможные обобшения теоремы 1.

Отметим также, что основные результаты этой статьи были, без доказательства, сформулированы в статье [7] (см. там теоремы 1 и 2 и следствия 1 и 2). 


\section{§3. Оценки сверху для модуля изменения и усредненного модуля}

Прежде всего заметим, что если число $\delta>0$ и натуральное число $N$ таковы, что $\delta \in\left[\pi / \lambda_{N}, \pi / \lambda_{N-1}\right)$, то из (8) следуют равенства: $N=N(\delta)$ и

$$
\begin{aligned}
\omega_{0}(\delta) & =w_{N-1} \delta+\pi \sum_{n=N}^{\infty} \lambda_{n}^{-1}\left(w_{n}-w_{n-1}\right) \\
& =\delta U\left(\frac{\pi}{\delta}\right)+\pi \sum_{n=N(\delta)}^{\infty} \lambda_{n}^{-1}\left(w_{n}-w_{n-1}\right) .
\end{aligned}
$$

В частности,

$$
\begin{aligned}
\omega_{0}\left(\frac{\pi}{\lambda_{N}}\right) & =\pi \lambda_{N}^{-1} w_{N-1}+\pi \sum_{n=N}^{\infty} \sum_{m=n}^{\infty}\left(\lambda_{m}^{-1}-\lambda_{m+1}^{-1}\right)\left(w_{n}-w_{n-1}\right) \\
& =\pi \sum_{m=N}^{\infty}\left(\lambda_{m}^{-1}-\lambda_{m+1}^{-1}\right) w_{m} .
\end{aligned}
$$

ДоказАТЕЛЬСТво ЛЕммы 1 . Пусть $\delta \in\left[\pi / \lambda_{N}, \pi / \lambda_{N-1}\right)$, где $N$ - натуральное число. Из $(25)$ и $(9)$ сразу имеем $\omega_{0}(\delta) \leqslant \delta U(\pi / \delta)+\pi a(\delta)$. Из $(26)$ и $(7)$ получаем

$$
\pi \sum_{m=N}^{\infty} \lambda_{m}^{-1} w_{m} \geqslant \omega_{0}\left(\pi / \lambda_{N}\right) \geqslant\left(1-\lambda^{-1}\right) \pi \sum_{m=N}^{\infty} \lambda_{m}^{-1} w_{m} \geqslant\left(1-\lambda^{-1}\right) \pi \sum_{m=N}^{\infty} a_{m} .
$$

Этим доказаны оценки (11) и оценка

$$
\omega_{0}(\delta) \geqslant \omega_{0}\left(\pi / \lambda_{N}\right) \geqslant\left(1-\lambda^{-1}\right) \pi a(\delta) .
$$

Отсюда и из $(25)$ следует $\delta U(\pi / \delta)+\pi a(\delta) \leqslant\left(1+\lambda(\lambda-1)^{-1}\right) \omega_{0}(\delta)$. Этим оценки (10), а значит, и (12), также доказаны.

Отметим, что из (25) и (26) сразу вытекает представление

$$
\omega_{0}(\delta)=\int_{0}^{\delta} U\left(\frac{\pi}{t}\right) d t \text { при всех } \delta \geqslant 0,
$$

которое удобно при оценивании $\omega_{0}$ в конкретных случаях.

ДОКАЗАТЕЛЬСТВО ВЕРХНЕЙ ОЦЕНКИ В (15). Пусть $n$ - произвольное натуральное число и $\left\{\left(\alpha_{k}, \beta_{k}\right)\right\}_{k=1}^{n}-$ произвольньй набор непересекаюшихся интервалов, лежаших в сегменте $I=[\alpha, \beta]$, где $\alpha<\beta$ и $\alpha_{k} \leqslant \beta_{k}$ при $k=1, \ldots, n$. Пусть $\Delta=|I|=\beta-\alpha, \gamma=\Delta / n$ и $N=N(\gamma)$. Обозначим $S(x)=\sum_{n=1}^{N-1} a_{n} \cos \left(\lambda_{n} x+\psi_{n}\right)$. Тогда при каждом $k=1, \ldots, n$ имеем

$$
\begin{aligned}
\left|f\left(\beta_{k}\right)-f\left(\alpha_{k}\right)\right| & \leqslant\left|S\left(\beta_{k}\right)-S\left(\alpha_{k}\right)\right|+2 \sum_{n=N}^{\infty} a_{n} \\
& =\left|\int_{\alpha_{k}}^{\beta_{k}} S^{\prime}(x) d x\right|+2 a(\gamma) \leqslant \int_{\alpha_{k}}^{\beta_{k}}\left|S^{\prime}(x)\right| d x+2 a(\gamma) .
\end{aligned}
$$


Следовательно,

$$
\sum_{k=1}^{n}\left|f\left(\beta_{k}\right)-f\left(\alpha_{k}\right)\right| \leqslant \int_{I}\left|S^{\prime}(x)\right| d x+2 n a(\gamma)
$$

Отсюда сразу следует, что

$$
\nu_{f}(n)_{I} \leqslant \int_{I}\left|S^{\prime}(x)\right| d x+2 n a(\gamma)
$$

Но (см. [8, гл. 5, теорема 8.20], [9, теорема 1])

$$
\begin{aligned}
\int_{I}\left|S^{\prime}(x)\right| d x & =\int_{I}\left|\sum_{n=1}^{N-1} a_{n} \lambda_{n} \sin \left(\lambda_{n} x+\psi_{n}\right)\right| d x \\
& \leqslant A(\lambda)\left(\Delta+\lambda_{1}^{-1}\right) w_{N-1}=\Delta A(\lambda) K U\left(\frac{\pi}{\gamma}\right),
\end{aligned}
$$

где $K=1+\left(\lambda_{1}|I|\right)^{-1}$. Поэтому из $(27),(8)$ и (12) получаем (напомним, что постоянная $A$ в каждом случае своя)

$$
\begin{aligned}
\nu_{f}(n)_{I} & \leqslant A(\lambda) K n \gamma U\left(\frac{\pi}{\gamma}\right)+2 n a(\gamma) \leqslant \max \left\{A(\lambda) K, \frac{2}{\pi}\right\} n \gamma L\left(\frac{\pi}{\gamma}\right) \\
& \leqslant A(\lambda)\left(1+\left(\lambda_{1}|I|\right)^{-1}\right) n \omega_{0}\left(\frac{|I|}{n}\right) .
\end{aligned}
$$

Этим верхняя оценка (15) доказана, причем постоянную в ней можно взять в виде

$$
A\left(\lambda, \lambda_{1}|I|\right)=A(\lambda)\left(1+\left(\lambda_{1}|I|\right)^{-1}\right) .
$$

ДОКАЗАТЕЛЬСТВО ВЕРХНЕЙ ОЦЕНКИ В (13). Поскольку При $\delta \geqslant 2 \Delta$ фунКция $\tau_{f}(\delta)_{I}$ преврашается в постоянную, то верхнюю оценку в (13) достаточно доказывать при $\delta \in(0,2 \Delta]$, где $\Delta=|I|$ - длина сегмента $I$. В этом случае существует натуральное $n$ такое, что $\delta \in(2 \Delta /(n+1), 2 \Delta / n]$. Тогда в силу (5) и уже доказанной верхней оценки в (15) имеем

$$
\tau_{f}(\delta)_{I} \leqslant 2 \tau_{f}\left(\frac{\Delta}{n}\right)_{I} \leqslant 2 n^{-1} \nu_{f}(n)_{I} \leqslant A\left(\lambda, \lambda_{1}|I|\right) \omega_{0}\left(\frac{\Delta}{n}\right) \leqslant A\left(\lambda, \lambda_{1}|I|\right) \omega_{0}(\delta) .
$$

Этим верхняя оценка в (13) полностью доказана, причем соответствуюшую постоянную можно взять в виде (28). 


\section{§4. Завершение доказательства теоремы 1}

Напомним, что $f(x)$ - это сумма ряда (6). При всех $\delta \geqslant 0$ рассмотрим функцию

$$
\underline{\omega}(\delta ; f)=\inf \max _{x \in J}|f(x)-c|,
$$

где нижняя грань берется по всем числам $c$ и всем сегментам $J$, длина которых равна $|J|=\delta$. Ясно, что при всех $\delta \geqslant 0$ имеют место представления

$$
\begin{aligned}
\underline{\omega}(\delta ; f) & =\inf _{x, c} \max _{t \in[0, \delta]}|f(x+t)-c|=\inf _{x, c} \max _{t \in[0, \delta]}|f(x-t)-c| \\
& =\inf _{x, c} \max _{|t| \leqslant \delta / 2}|f(x+t)-c| \\
& =\inf _{x, c} \max _{|t| \leqslant \delta / 2}\left|c+\sum_{n=1}^{\infty} a_{n} \cos \left(\lambda_{n} t+\lambda_{n} x+\psi_{n}\right)\right|,
\end{aligned}
$$

где каждая из нижних граней берется по всем числам $c$ и $x$. Поскольку для любого сегмента $J$ колебание

$$
\Omega_{f}(J)=\max _{x \in J} f(x)-\min _{x \in J} f(x)=2 \min _{c} \max _{x \in J}|f(x)-c|,
$$

то из определения (29) очевидно равенство

$$
\underline{\omega}(\delta ; f)=\frac{1}{2} \inf \Omega_{f}(J),
$$

где нижняя грань берется по всем сегментам $J$, длина которых равна $|J|=\delta$. Отсюда легко вытекает

Лемма 2. Для любого сегмента $I,|I|>0$, при всех $\delta \in[0,|I|]$ верна оценка: $\tau_{f}(\delta)_{I} \geqslant \underline{\omega}(\delta ; f)$.

ДокАЗАТЕЛЬСТво. Из (31) и (4) при $\delta \in[0,|I|]$ имеем

$$
2 \tau_{f}(\delta)_{I} \geqslant \tau_{f}(2 \delta)_{I}=\frac{1}{|I|} \int_{I} \Omega_{f}([x-\delta, x+\delta] \cap I) d x \geqslant 2 \underline{\omega}(\delta ; f) .
$$

Этим лемма 2 доказана.

При всех $\delta \geqslant 0$ рассмотрим также функцию

$$
\theta(\delta)=\inf \max _{|t| \leqslant \delta / 2}\left|c+\sum_{n=1}^{\infty} a_{n} \cos \left(\lambda_{n} t+\varphi_{n}\right)\right|
$$

где нижняя грань берется по всем числам $c$ и $\left\{\varphi_{n}\right\}_{n=1}^{\infty}$. Здесь последовательности $\left\{a_{n}\right\}_{n=1}^{\infty}$ и $\left\{\lambda_{n}\right\}_{n=1}^{\infty}$ те же, что и в (6). Из представления (30) сразу получаем, что

$$
\underline{\omega}(\delta ; f) \geqslant \theta(\delta) \text { при всех } \delta \geqslant 0 .
$$

Важной частью доказательства теоремы 1 является 
Лемма 3. При всех $\delta>0$ верна оценка:

$$
\omega_{0}(\delta) \leqslant A(\lambda)\left(\theta(\delta)+\delta U\left(\frac{\pi}{\delta}\right)\right)
$$

ДоКАЗАТЕЛЬСТво. При любом $h \geqslant 0$ очевидно тождество

$$
f(x+h)-2 f(x)+f(x-h)=-\sum_{n=1}^{\infty} 4 \sin ^{2}\left(\frac{\lambda_{n} h}{2}\right) a_{n} \cos \left(\lambda_{n} x+\psi_{n}\right) .
$$

Пусть положительное число $\varepsilon$ пока произвольно. При каждом натуральном $N$, полагая $\delta=\varepsilon\left(2 \lambda_{N}\right)^{-1}$, получаем

$$
\begin{aligned}
& \max _{|x| \leqslant \delta}|f(x+h)-2 f(x)+f(x-h)|+\sum_{n=1}^{N-1} 4 a_{n} \sin ^{2}\left(\frac{\lambda_{n} h}{2}\right) \\
& \quad \geqslant \max _{|x| \leqslant \delta}\left|\sum_{n=N}^{\infty} 4 \sin ^{2}\left(\lambda_{n} h / 2\right) a_{n} \cos \left(\lambda_{n} x+\psi_{n}\right)\right| \\
& \quad \geqslant A^{-1}(\lambda, \varepsilon) \sum_{n=N}^{\infty} 4 a_{n} \sin ^{2}\left(\frac{\lambda_{n} h}{2}\right)
\end{aligned}
$$

где последнее неравенство следует из варианта известного неравенства СидонаЗигмунда, доказанного автором в [10, теорема 2]. Следовательно,

$$
4 \max _{|t| \leqslant \delta+h}|f(t)|+\sum_{n=1}^{N-1} a_{n} \lambda_{n}^{2} h^{2} \geqslant A^{-1}(\lambda, \varepsilon) \sum_{n=N}^{\infty} 4 a_{n} \sin ^{2}\left(\frac{\lambda_{n} h}{2}\right) .
$$

Возьмем здесь нижнюю грань по всем числам $a$ и $\left\{\psi_{n}\right\}_{n=1}^{\infty}$ (см. (6)). Тогда получим неравенство

$$
4 \theta\left(\varepsilon \lambda_{N}^{-1}+2 h\right)+\sum_{n=1}^{N-1} a_{n} \lambda_{n}^{2} h^{2} \geqslant A^{-1}(\lambda, \varepsilon) \sum_{n=N}^{\infty} 4 a_{n} \sin ^{2}\left(\frac{\lambda_{n} h}{2}\right),
$$

которое справедливо при всех натуральных $N$ и действительных $h \geqslant 0$. Положим $h=\varepsilon \lambda_{N}^{-1} t$, где $t \in[0,1]$, и проинтегрируем обе части полученного неравенства по $t$ от 0 до 1 . Поскольку функция $\theta$ не убывает на $[0, \infty)$, то в результате получаем

$$
\begin{aligned}
4 \theta\left(3 \varepsilon \lambda_{N}^{-1}\right)+\frac{1}{3} \varepsilon^{2} \lambda_{N}^{-2} \sum_{n=1}^{N-1} a_{n} \lambda_{n}^{2} & \geqslant A^{-1}(\lambda, \varepsilon) \sum_{n=N}^{\infty} 4 a_{n} \int_{0}^{1} \sin ^{2}\left(\frac{\varepsilon \lambda_{n}}{2 \lambda_{N}} t\right) d t \\
& \geqslant 4 A^{-1}(\lambda, \varepsilon)\left(\inf _{v \geqslant \varepsilon / 2} \int_{0}^{1} \sin ^{2}(v t) d t\right) \sum_{n=N}^{\infty} a_{n} .
\end{aligned}
$$

Отсюда, беря $\varepsilon=\pi / 3$ и деля все на 4 , приходим к оценке

$$
\theta\left(\frac{\pi}{\lambda_{N}}\right)+\pi^{2} \lambda_{N}^{-2} \sum_{n=1}^{N-1} a_{n} \lambda_{n}^{2} \geqslant A^{-1}(\lambda) \sum_{n=N}^{\infty} a_{n}
$$


которая справедлива при всех натуральных $N$. Возьмем произвольное $\delta>0$ и (см. (8)) положим в полученной оценке $N=N(\delta)$. Сразу получаем оценку

$$
\theta(\delta)+\delta^{2} \sum_{n=1}^{N(\delta)-1} a_{n} \lambda_{n}^{2} \geqslant A^{-1}(\lambda) a(\delta)
$$

Но в силу (7)

$$
\sum_{n=1}^{N-1} a_{n} \lambda_{n}^{2} \leqslant w_{N-1}\left(\sum_{n=1}^{N-1} \lambda_{n}^{2}\right)^{1 / 2} \leqslant w_{N-1} \lambda_{N-1}\left(1-\lambda^{-2}\right)^{-1 / 2} .
$$

Поэтому

$$
\delta \sum_{n=1}^{N(\delta)-1} a_{n} \lambda_{n}^{2} \leqslant \pi\left(1-\lambda^{-2}\right)^{-1 / 2} U\left(\frac{\pi}{\delta}\right)
$$

Отсюда, из (34) и (10) имеем

$$
\omega_{0}(\delta) \leqslant \pi a(\delta)+\delta U\left(\frac{\pi}{\delta}\right) \leqslant A(\lambda) \theta(\delta)+A(\lambda) \delta U\left(\frac{\pi}{\delta}\right)
$$

Оценка (33) доказана.

Пусть теперь $I=[\alpha, \beta]$ - произвольный сегмент и $\Delta=|I|=\beta-\alpha>0$ - его длина. Завершим доказательство соотношений (13), (14) и (15).

ДОКАЗАТЕЛЬСТВО НИЖНЕЙ ОЦЕНКИ В (13). Пусть сначала $\delta \in(0, \Delta]$. Тогда в силу (4) при любом $h \in[0, \delta]$ верна оценка

$$
\begin{aligned}
\Delta \tau_{f}(\delta)_{I} & \geqslant \int_{\alpha+h / 2}^{\beta-h / 2} \Omega_{f}([x-h / 2, x+h / 2]) d x \\
& \geqslant \int_{\alpha+h / 2}^{\beta-h / 2}|f(x+h / 2)-f(x-h / 2)| d x \\
& =\int_{\alpha+h / 2}^{\beta-h / 2}\left|\sum_{n=1}^{\infty} 2 a_{n} \sin \left(\frac{\lambda_{n} h}{2}\right) \sin \left(\lambda_{n} x+\psi_{n}\right)\right| d x
\end{aligned}
$$

Отсюда при $h=\delta / 2$ по оценке из [9, теорема 2] имеем

$$
\begin{aligned}
\tau_{f}(\delta)_{I} & \geqslant \frac{1}{\Delta} \int_{\alpha+\Delta / 4}^{\beta-\Delta / 4}\left|\sum_{n=1}^{\infty} 2 a_{n} \sin \left(\frac{\lambda_{n} \delta}{4}\right) \sin \left(\lambda_{n} x+\psi_{n}\right)\right| \\
& \geqslant A^{-1}\left(\lambda, \Delta \lambda_{1}\right)\left(\sum_{n=1}^{\infty} a_{n}^{2} \sin ^{2}\left(\frac{\lambda_{n} \delta}{4}\right)\right)^{1 / 2} \\
& \geqslant A^{-1}\left(\lambda, \Delta \lambda_{1}\right)\left(\sum_{n=1}^{N(\delta)-1} a_{n}^{2}\left(\frac{\lambda_{n} \delta}{2 \pi}\right)^{2}\right)^{1 / 2}
\end{aligned}
$$


Следовательно, $\tau_{f}(\delta)_{I} \geqslant A^{-1}\left(\lambda, \Delta \lambda_{1}\right) \delta U(\pi / \delta)$. Отсюда, из леммы 2 и оценки $(32)$ получаем оценку

$$
A\left(\lambda, \Delta \lambda_{1}\right) \tau_{f}(\delta)_{I} \geqslant \theta(\delta)+\delta U\left(\frac{\pi}{\delta}\right)
$$

Вместе с леммой 3 это дает нижнюю оценку в (13) при $\delta \in(0, \Delta]$. Теперь заметим, что по варианту неравенства Сидона-Зигмунда [10, теорема 2] для любых чисел $c$ и $\left\{\varphi_{n}\right\}_{n=1}^{\infty}$ верна оценка

$$
\max _{|t| \leqslant \Delta / 2}\left|c+\sum_{n=1}^{\infty} a_{n} \cos \left(\lambda_{n} t+\varphi_{n}\right)\right| \geqslant A^{-1}\left(\lambda, \Delta \lambda_{1}\right) \sum_{n=1}^{\infty} a_{n},
$$

т.e.

$$
A\left(\lambda, \Delta \lambda_{1}\right) \theta(\Delta) \geqslant \sum_{n=1}^{\infty} a_{n}
$$

В силу (8) и (9) очевидна оценка

$$
\omega_{0}(\delta) \leqslant \omega_{1}(\delta) \text { при всех } \delta \geqslant 0 .
$$

Поэтому при всех $\delta \geqslant \Delta$ получаем

$$
A\left(\lambda, \Delta \lambda_{1}\right) \theta(\Delta) \geqslant \pi \sum_{n=1}^{\infty} a_{n} \geqslant \omega_{1}(\delta) \geqslant \omega_{0}(\delta) .
$$

Отсюда, из леммы 2 и оценки (32) при $\delta \geqslant \Delta$ имеем

$$
\tau_{f}(\delta)_{I} \geqslant \tau_{f}(\Delta)_{I} \geqslant \theta(\Delta) \geqslant A^{-1}\left(\lambda, \Delta \lambda_{1}\right) \omega_{0}(\delta)
$$

Этим нижняя оценка в (13) доказана при всех $\delta \geqslant 0$.

ДОКАЗАТЕЛЬСТВо нИЖНЕЙ ОцЕНКИ В (15). Из уже доказанной нижней оценки в (13) и оценки (5) при каждом натуральном $n$ имеем

$$
n^{-1} \nu_{f}(n)_{I} \geqslant \tau_{f}\left(\frac{\Delta}{n}\right)_{I} \geqslant A^{-1}\left(\lambda, \Delta \lambda_{1}\right) \omega_{0}\left(\frac{\Delta}{n}\right)
$$

Оценка (15) полностью доказана.

ДоказАТЕЛЬСтво оценок (14). Нижняя оценка в (14) - это оценка (5). Поскольку для каждого натурального $n$ из верхней оценки (15) и нижней оценки (13) имеем

$$
n^{-1} \nu_{f}(n)_{I} \leqslant A\left(\lambda, \Delta \lambda_{1}\right) \omega_{0}\left(\frac{\Delta}{n}\right) \leqslant A\left(\lambda, \Delta \lambda_{1}\right) \tau_{f}\left(\frac{\Delta}{n}\right)_{I}
$$

то верна и верхняя оценка (14). Теорема 1 полностью доказана. 


\section{§5. Следствия из теоремы 1}

ДокАЗАТЕЛЬСТво СлЕДСТвИЯ 1. Пусть $p>0$ и $\gamma \in(1, p+1]$. По оценкам (15) и (16) $f \in \operatorname{ch}_{\gamma}^{p}$ на $I$ тогда и только тогда, когда

$$
\sum n^{-\gamma}\left(n \omega_{0}\left(\frac{\pi}{n}\right)\right)^{p}<\infty, \quad \text { т.е. } \int_{1}^{\infty} y^{-\gamma}\left(y \omega_{0}\left(\frac{\pi}{y}\right)\right)^{p} d y<\infty .
$$

Последнее условие в силу оценок (12) эквивалентно условию

$$
\int_{1}^{\infty} y^{-\gamma}(L(y))^{p} d y<\infty .
$$

Поскольку при $N=1,2, \ldots$ и $y \in\left(\lambda_{N-1}, \lambda_{N}\right]$ функция

$$
L(y)=w_{N-1}+y \sum_{n=N}^{\infty} a_{n},
$$

то $f \in \operatorname{ch}_{\gamma}^{p}$ на $I$ тогда и только тогда, когда выполнены следуюшие два условия

$$
\sum_{N=2}^{\infty}\left(w_{N-1}\right)^{p} \int_{\lambda_{N-1}}^{\lambda_{N}} y^{-\gamma} d y<\infty \text { и } \sum_{N=2}^{\infty}\left(\sum_{n=N}^{\infty} a_{n}\right)^{p} \int_{\lambda_{N-1}}^{\lambda_{N}} y^{p-\gamma} d y<\infty .
$$

В силу (7) интеграл в первом условии имеет порядок $\lambda_{N-1}^{1-\gamma}$, а интеграл из второго условия имеет порядок $\lambda_{N}^{p+1-\gamma}$ при $\gamma<p+1$ и $\ln \left(\lambda_{N} / \lambda_{N-1}\right)$ при $\gamma=p+1$. Ясно, что как из первого, так и из второго условий (36) сразу вытекает условие (17). Если $\gamma<p+1$, то условия (36) преврашаются в условия

$$
\sum_{N=1}^{\infty} \lambda_{N}^{1-\gamma}\left(\sum_{n=1}^{N} a_{n}^{2} \lambda_{n}^{2}\right)^{p / 2}<\infty \text { и } \sum_{N=1}^{\infty} \lambda_{N}^{p+1-\gamma}\left(\sum_{n=N}^{\infty} a_{n}\right)^{p}<\infty
$$

причем первое условие (36) эквивалентно первому условию (37) и при $\gamma=p+1$.

Докажем сначала, что из условия (17) вытекает первое условие (37). Действительно, пусть положительное $\delta$ таково, что $\delta p / 2<\gamma-1$. Тогда если $p>2$, то при натуральных $N$ по неравенству Гёльдера имеем

$$
\begin{aligned}
\left(\sum_{n=1}^{N} a_{n}^{2} \lambda_{n}^{2}\right)^{p / 2} & \leqslant \sum_{n=1}^{N}\left(a_{n}^{2} \lambda_{n}^{2-\delta}\right)^{p / 2}\left(\sum_{n=1}^{N} \lambda_{n}^{\delta p /(p-2)}\right)^{(p-2) / 2} \\
& \leqslant \lambda_{N}^{\delta p / 2}\left(\sum_{n=1}^{N} a_{n}^{p} \lambda_{n}^{p-\delta p / 2}\right)\left(\sum_{k=0}^{\infty} \lambda^{-k \delta p /(p-2)}\right)^{(p-2) / 2}
\end{aligned}
$$

Если $p \leqslant 2$, то при натуральных $N$ получаем

$$
\left(\sum_{n=1}^{N} a_{n}^{2} \lambda_{n}^{2}\right)^{p / 2} \leqslant \sum_{n=1}^{N} a_{n}^{p} \lambda_{n}^{p} \leqslant \lambda_{N}^{\delta p / 2} \sum_{n=1}^{N} a_{n}^{p} \lambda_{n}^{p-\delta p / 2} .
$$


В любом случае первое условие (37) будет выполнено, если

$$
\sum_{N=1}^{\infty} \lambda_{N}^{1-\gamma+\delta p / 2} \sum_{n=1}^{N} a_{n}^{p} \lambda_{n}^{p-\delta p / 2}=\sum_{n=1}^{\infty}\left(\sum_{N=n}^{\infty} \lambda_{N}^{1-\gamma+\delta p / 2}\right) \lambda_{n}^{p-\delta p / 2} a_{n}^{p}<\infty
$$

Последнее же условие немедленно вытекает из (17). Таким образом, условие (17) эквивалентно первому условию (36).

Докажем теперь, что при $\gamma<p+1$ из условия (17) следует второе условие (37). Для этого возьмем такое положительное $\delta$, что $\delta p<p+1-\gamma$. Тогда если $p>1$, то при натуральных $N$ имеем

$$
\begin{aligned}
\left(\sum_{n=N}^{\infty} a_{n}\right)^{p} & \leqslant \sum_{n=N}^{\infty}\left(a_{n} \lambda_{n}^{\delta}\right)^{p}\left(\sum_{n=N}^{\infty} \lambda_{n}^{-\delta p /(p-1)}\right)^{p-1} \\
& \leqslant \lambda_{N}^{-\delta p}\left(\sum_{n=N}^{\infty} a_{n}^{p} \lambda_{n}^{\delta p}\right)\left(\sum_{k=0}^{\infty} \lambda^{-k \delta p /(p-1)}\right)^{p-1}
\end{aligned}
$$

Если же $p \leqslant 1$, то при натуральных $N$ получаем

$$
\left(\sum_{n=N}^{\infty} a_{n}\right)^{p} \leqslant \sum_{n=N}^{\infty} a_{n}^{p} \leqslant \lambda_{N}^{-\delta p} \sum_{n=N}^{\infty} a_{n}^{p} \lambda_{n}^{\delta p}
$$

В любом случае второе условие (37) будет выполнено, если

$$
\sum_{N=1}^{\infty} \lambda_{N}^{p+1-\gamma-\delta p} \sum_{n=N}^{\infty} a_{n}^{p} \lambda_{n}^{\delta p}=\sum_{n=1}^{\infty}\left(\sum_{N=1}^{n} \lambda_{N}^{p+1-\gamma-\delta p}\right) \lambda_{n}^{\delta p} a_{n}^{p}<\infty
$$

Последнее же условие немедленно вытекает из (17). Утверждение 1) следствия 1 доказано.

Пусть теперь $\gamma=p+1$. Тогда второе условие (36) совпадает с условием (18). Докажем, что из (18) следует и первое условие (36). Действительно, из (18), т.е. второго условия (36), вытекает, что $\sum a_{N}^{p} \ln \left(\lambda_{N} / \lambda_{N-1}\right)<\infty$. В частности, выполнено условие (17), а значит, как уже доказано выше, выполнено эквивалентное ему первое условие (36). Утверждение 2) следствия 1 также доказано.

Пусть далее $p=1$ и $\gamma=2$. Тогда условие (18) совпадает с условием

$$
\sum_{N=2}^{\infty}\left(\sum_{n=N}^{\infty} a_{n}\right) \ln \frac{\lambda_{N}}{\lambda_{N-1}}<\infty
$$

Это последнее условие, очевидно, совпадает с условием (19). Следствие 1 полностью доказано. 
ДОКАЗАТЕЛЬСТво СЛЕДСТВИЯ 2. Пусть $\omega(\delta ; f)=\sup \left\{\left|f\left(x_{1}\right)-f\left(x_{2}\right)\right|\right.$ : $\left.\left|x_{1}-x_{2}\right| \leqslant \delta\right\}, \delta \geqslant 0,-$ модуль непрерьвности функции $f$ на всей прямой. Тогда (см. [11, формулы (26), (27) и (33)])

$$
A^{-1}(\lambda) \omega_{1}(\delta) \leqslant \omega(\delta ; f) \leqslant \omega_{1}(\delta) \text { при всех } \delta \geqslant 0 .
$$

Известно также, что (см. [10, теорема 3$]$ )

$$
A^{-1}\left(\lambda, \Delta \lambda_{1}\right) \omega(\delta ; f) \leqslant \omega(\delta ; f)_{I} \leqslant \omega(\delta ; f) \text { при всех } \delta \geqslant 0,
$$

где $\Delta=|I|$ - длина сегмента $I$. Из теоремы 1 и оценок (38) и (39) сразу вытекает, что утверждение 1) следствия 2 эквивалентно утверждению: сушествует такая положительная постоянная $C$, что

$$
\omega_{1}\left(\frac{\Delta}{n}\right) \leqslant C \omega_{0}\left(\frac{\Delta}{n}\right) \text { при } n \geqslant 1 .
$$

Это последнее утверждение, очевидно, эквивалентно утверждению 2) следствия 2. Отметим, что при $\delta \geqslant 0$ верны представления

$$
\omega_{1}(\delta)=\delta T\left(\frac{\pi}{\delta}\right)+\pi a(\delta)=\pi \delta \int_{\delta}^{\infty} t^{-2} a(t) d t=\int_{0}^{\delta} T\left(\frac{\pi}{t}\right) d t .
$$

Поскольку функции $\omega_{1}$ и $\omega_{0}$ линейны на сегментах $\left[\pi / \lambda_{n}, \pi / \lambda_{n-1}\right], n \geqslant 2$, и постоянны на промежутке $\left[\pi / \lambda_{1}, \infty\right)$, то $(21)$ эквивалентно соотношению $\omega_{1}\left(\pi / \lambda_{n}\right) \leqslant$ $C_{2} \omega_{0}\left(\pi / \lambda_{n}\right)$ при $n \geqslant 1$. При натуральном $n$ в силу (40) и леммы 1 верны соотношения:

$$
\omega_{1}\left(\frac{\pi}{\lambda_{n}}\right)=\pi \lambda_{n}^{-1} v_{n}+\pi \sum_{k=n+1}^{\infty} a_{k}
$$

и

$$
\omega_{0}\left(\frac{\pi}{\lambda_{n}}\right) \leqslant \pi \lambda_{n}^{-1} w_{n-1}+\pi \sum_{k=n}^{\infty} a_{k} \leqslant A(\lambda) \omega_{0}\left(\frac{\pi}{\lambda_{n}}\right) .
$$

Поэтому утверждение 2) следствия 2 эквивалентно утверждению: существует такая положительная постоянная $C$, что

$$
v_{n} \leqslant C\left(w_{n-1}+\lambda_{n} \sum_{k=n}^{\infty} a_{k}\right) \text { при } n \geqslant 1 .
$$

Поскольку $w_{n} \leqslant w_{n-1}+a_{n} \lambda_{n}$, то последнее утверждение эквивалентно утверждению 3 ) следствия 2 . При каждом натуральном $n$ имеем

$$
C_{3} w_{n} \leqslant C_{3} v_{n}^{1 / 2}\left(\max _{k \leqslant n} a_{k} \lambda_{k}\right)^{1 / 2} \leqslant \frac{1}{2} v_{n}+\frac{1}{2} C_{3}^{2} \max _{k \leqslant n}\left(a_{k} \lambda_{k}\right) .
$$

Поэтому из (22) вытекает, что при всех натуральных $n$ верна оценка

$$
v_{n} \leqslant \frac{1}{2} v_{n}+\frac{1}{2} C_{3}^{2} \max _{k \leqslant n}\left(a_{k} \lambda_{k}\right)+C_{3} \lambda_{n} \sum_{k=n}^{\infty} a_{k},
$$


т.е. верно утверждение 4) следствия 2. Обратно, из утверждения 4), очевидно, сразу вытекает утверждение 3) следствия 2. Таким образом, условия (20), (21), (22) и (23) эквивалентны между собой. Этим следствие 2 полностью доказано.

Из оценки (3) и следствия 2 вытекает, что для функции (6) соотношение

$$
\nu_{f}(n)_{I} \asymp n \omega\left(\frac{|I|}{n} ; f\right)_{I} \text { при } n \geqslant 1
$$

верно тогда и только тогда, когда для некоторой положительной постоянной $C_{4}$ вьполнена оценка (23).

ДоКАЗАТЕЛЬСТВО СЛЕДСТВИЯ 3. Если последовательность $\nu=\{\nu(n)\}_{n=0}^{\infty}$ ограничена, то, очевидно, в качестве $f(x)$ можно взять любую функцию (6), у которой $0<\sum_{n=1}^{\infty} a_{n} \lambda_{n}<\infty$. Поэтому будем предполагать, что последовательность $\nu$ неограничена. Поскольку последовательность $\nu$ не убывает, то $\nu(n) \rightarrow \infty$ при $n \rightarrow \infty$ По условию последовательность $\{\nu(n) / n\}_{n=0}^{\infty}$ не возрастая стремится к нулю. Положим $\lambda_{1}=1$. Если числа $\lambda_{1}, \ldots, \lambda_{m}$ при некотором натуральном $m$ уже выбраны, то обозначим через $\lambda_{m+1}$ наименьшее натуральное число такое, что $\nu\left(\lambda_{m+1}\right)>2 \nu\left(\lambda_{m}\right)$. Поскольку $\nu\left(2 \lambda_{m}\right) \leqslant 2 \nu\left(\lambda_{m}\right)$, то $\lambda_{m+1}>2 \lambda_{m}$ при всех натуральных $m$. Положим

$$
a_{n}=\nu\left(\lambda_{n}\right) \lambda_{n}^{-1}-\nu\left(\lambda_{n+1}\right) \lambda_{n+1}^{-1} \text { при } n \geqslant 1 .
$$

Тогда $a_{n} \geqslant 0$ при $n \geqslant 1$ и $\sum a_{n}<\infty$. Числа $a$ и $\left\{\psi_{n}\right\}_{n=1}^{\infty}$ возьмем произвольньм образом. Рассмотрим соответствуюшую функцию (6). При натуральных $n$ имеем

$$
\sum_{k=n}^{\infty} a_{k}=\nu\left(\lambda_{n}\right) \lambda_{n}^{-1}
$$

и

$$
v_{n}=\sum_{k=1}^{n} a_{k} \lambda_{k} \leqslant \sum_{k=1}^{n} \nu\left(\lambda_{k}\right) \leqslant 2 \nu\left(\lambda_{n}\right)=2 \lambda_{n} \sum_{k=n}^{\infty} a_{k} .
$$

Поэтому выполнено утверждение 3 ) следствия 2. По следствию 2 и оценкам (3), $(38)$ и (39) на любом сегменте $I=[\alpha, \beta], \alpha<\beta$, имеем

$$
\nu_{f}(n)_{I} \asymp n \omega\left(\frac{|I|}{n} ; f\right)_{I} \asymp n \omega_{1}\left(\frac{\pi}{n}\right) .
$$

Если $\lambda_{m} \leqslant n<\lambda_{m+1}$ при некотором натуральном $m$, то в силу (8), (41) и (42)

$$
n \omega_{1}\left(\frac{\pi}{n}\right)=\pi v_{m}+\pi n \sum_{k=m+1}^{\infty} a_{k} \leqslant 2 \pi \nu\left(\lambda_{m}\right)+\pi n \lambda_{m+1}^{-1} \nu\left(\lambda_{m+1}\right) \leqslant 3 \pi \nu(n)
$$

И

$$
\begin{aligned}
n \omega_{1}\left(\frac{\pi}{n}\right) & \geqslant \pi v_{m}+\pi \lambda_{m} \sum_{k=m+1}^{\infty} a_{k} \\
& \geqslant \pi \lambda_{m} \sum_{k=m}^{\infty} a_{k}=\pi \nu\left(\lambda_{m}\right) \geqslant \frac{\pi}{2} \nu\left(\lambda_{m+1}-1\right) \geqslant \frac{\pi}{2} \nu(n) .
\end{aligned}
$$


Следовательно,

$$
\frac{\pi}{2} \nu(n) \leqslant n \omega_{1}\left(\frac{\pi}{n}\right) \leqslant 3 \pi \nu(n) \text { при всех } n \geqslant 1
$$

и соотношения (24) доказаны.

ЗАмЕчаниЕ 1 . Пусть $k$-произвольное натуральное число и $p \in[1, \infty)$. Тогда для функции (6) разность

$$
\Delta_{h}^{k} f(x)=\sum_{j=0}^{k}(-1)^{k-j} C_{k}^{j} f(x+k h)
$$

при всех $h$ представима в виде

$$
\Delta_{h}^{k} f(x)=\sum_{n=1}^{\infty} a_{n}\left(2 \sin \left(\frac{\lambda_{n} h}{2}\right)\right)^{k} \cos \left(\lambda_{n} x+\frac{\lambda_{n} k h}{2}+\psi_{n}+\frac{\pi k}{2}\right) .
$$

Пусть $I=[\alpha, \beta], \alpha<\beta,-$ произвольный сегмент и число $\delta \geqslant 0$. Рассмотрим определенную при всех $x$ функцию

$$
\omega_{k}(f ; \delta, x)=\max \left\{\left|\Delta_{h}^{k} f(t)\right|\right\},
$$

где максимум берется по всем $t$ и $h$ таким, что $t, t+k h \in[x-k \delta / 2, x+k \delta / 2] \cap I$. Эта функция(см. [3, раздел 1.3]) называется локальным модулем гладкости порядка $k$ функции $f$ в точке $x$. При всех $\delta \geqslant 0$ усредненный модуль порядка $k$ функции $f$ определяется формулой

$$
\tau_{k}(f ; \delta)_{L^{p}(I)}=\left(\frac{1}{|I|} \int_{I}\left(\omega_{k}(f ; \delta, x)\right)^{p} d x\right)^{1 / p} .
$$

Отметим, что в этих обозначениях функция (4) совпадает с $\tau_{1}(f ; \delta)_{L^{1}(I)}$. Рассуждения, аналогичные использованньм при доказательстве теоремы 1 , позволяют получить порядковые оценки и для усредненного модуля порядка $k$ функции (6), т.е. верна

ТЕОРема 2. Для любых натурального $k, p \in[1, \infty)$ и любого сегмента $I=[\alpha, \beta], \alpha<\beta$, для функции (6) при всех $\delta>0$ справедливо порядковое соотношение

$$
\tau_{k}(f ; \delta)_{L^{p}(I)} \asymp \delta^{k}\left(\sum_{n=1}^{N(\delta)-1} a_{n}^{2} \lambda_{n}^{2 k}\right)^{1 / 2}+\pi^{k} \sum_{n=N(\delta)}^{\infty} a_{n},
$$

причем соответствующие этому соотношению положстельные постояннъе мохсно взять зависящими только от $p, \lambda, \lambda_{1}|I| u k$.

Отметим, что в случае $p=\infty$ правая часть формулы (43) дает модуль непрерывности порядка $k$ функции $f$ на сегменте $I$, т.е.

$$
\omega_{k}(f ; \delta)_{I}=\max \left\{\left|\Delta_{h}^{k} f(x)\right|: x, x+k h \in I\right\} .
$$

В этом случае (см. [10], [11]), при всех $\delta \geqslant 0$ верно порядковое соотношение

$$
\omega_{k}(f ; \delta)_{I} \asymp \sum_{n=1}^{\infty} a_{n} \min \left(\delta^{k} \lambda_{n}^{k}, \pi^{k}\right),
$$

причем соответствуюшие этому соотношению положительные постоянные можно взять зависяшими только от $\lambda, \lambda_{1}|I|$ и $k$. 


\section{Список литературы}

1. Чантурия 3. А. Модуль изменения и его применение в теории рядов Фурье // Докл. АН CCCP. 1974. T. 214. № 1. C. 63-66.

2. Севастьянов E. A. Кусочно-монотонная аптроксимация и $\Phi$-вариации // Anal. Math. 1975. T. 1. № 2. C. 141-164.

3. Сендов Б., Попов В. Усредненные модули гладкости. М.: Мир, 1988.

4. Долженко E. П., Севастьянов $E$. А. О приближении функций в хаусдорфовой метрике посредством кусочно-монотонных (в частности, рационалњных) функций // Матем. сб. 1976. T. 101. № 4. C. 508-541.

5. Севастьянов $E$. А. Средний модуль колебания и кусочно-монотонная аппроксимация // Матем. заметки. 1982. Т. 31. №6. С. 867-876.

6. Avdispahic $M$. Concepts of generalized bounded variation and the theory of Fourier series // Internat. J. Math. Math. Sci. 1986. V. 9. № 2. P. 223-244.

7. Белов А.С. Лакунарные тригонометрические ряды и функции обобщенной ограниченной вариации // Сообщения АН ГССР. 1989. Т. 134. №3. С. 49-52.

8. Зигмунд А. Тригонометрические ряды. Т. 1. М.: Мир, 1965.

9. Belov A.S. On some local properties of the sum of lacunary trigonometric series // Anal. Math. 1988. V. 14. №1. P. 65-97.

10. Белов A. С. О неравенстве Сидона-Зигмунда в теории лакунарных тригонометрических рядов // Матем. заметки. 1981. Т. 30. № 4. С. 502-515.

11. Белов А. С. О сумме лакунарного ряда // Труды ММО. 1975. Т. 33. С. 107-153.

Ивановский государственный университет

Поступила в редакцию 20.02 .1998 